\title{
Aufschluß von Pappel-Industrieholz nach dem Sulfatverfahren
}

B. Dix; E. Roffael, Wilhelm-Klauditz-Institut, Fraunhofer-Arbeitsgruppe für Holzforschung Bienroder Weg 54 E, 3300 Braunschweig

Subject: Sulfate pulps from poplar wood

Material und Methode: Hackschnitzel aus 14-15jährigem, entrindetem Pappelholz der Klone Muhle-Larsen (ML) und Androscoggin (AS) eines Standortes (Danndorf) wurden nach dem Sulfatverfahren aufgeschlossen:

Aufschluß:

Aufheizdauer:

Aufschlußdauer bei $\mathrm{T}_{\max }$

$\mathrm{NaOH}$ /atro Holz:

$\mathrm{Na}_{2}$ S/atro Holz:

$\mathrm{Na}_{2} \mathrm{CO}_{3} /$ atro Holz:

Flottenverhältnis (Holz:Lauge)

Aus den ungebleichten Zellstoffen wurden Prüfblätter angefertigt und deren wichtigsten physikalisch-technologischen Eigenschaften bestimmt (Tabelle)

\begin{tabular}{|c|c|c|c|c|c|}
\hline \multirow{3}{*}{$\begin{array}{l}\text { Zellstoff- } \\
\text { cigen- } \\
\text { schaft }\end{array}$} & \multirow{3}{*}{$\begin{array}{l}\text { Ein- } \\
\text { heit }\end{array}$} & \multicolumn{4}{|l|}{ Zellstoff } \\
\hline & & \multicolumn{2}{|l|}{$\begin{array}{l}\text { Klon } \\
\text { ML }\end{array}$} & \multicolumn{2}{|l|}{$\begin{array}{l}\text { Klon } \\
\text { AS }\end{array}$} \\
\hline & & A & $\mathrm{B}$ & $\mathrm{A}$ & B \\
\hline Mahldauer & $\min$ & 15 & 15 & 15 & 15 \\
\hline Mahlgrad & SR & 21 & 22 & 23 & 24 \\
\hline Reißlänge & & 4.28 & 6.69 & 4,06 & 6,83 \\
\hline $\begin{array}{l}\text { Fortreiß- } \\
\text { festigkeit }\end{array}$ & $\frac{\mathrm{mN} \cdot \mathrm{m}}{\mathrm{m}}$ & 1098 & 1547 & 861 & 1434 \\
\hline Falzfestigkeit & - & 79 & 1394 & 42 & 932 \\
\hline $\begin{array}{l}\text { Bersi- } \\
\text { festigkeil }\end{array}$ & $\frac{\mathrm{kPa} \cdot \mathrm{m}^{2}}{\mathrm{~g}}$ & 2,58 & 4.73 & 2,32 & 4,65 \\
\hline Weißgrad & $\%$ & 40,2 & 36,0 & 40,0 & 36,8 \\
\hline Kappa-Zahl & & 9,8 & 15,5 & 10,4 & 17,2 \\
\hline Ausbeute & $\%$ & 52,6 & 50,8 & 51.5 & 50,9 \\
\hline
\end{tabular}

Resultate: 1. Die Aufschlußbedingungen beeinflussen deutlich die Eigenschaften der Zellstoffe aus Pappelholz. Bei niedriger Aufschlußtemperatur und längerer Aufschlußdauer (Aufschluß B) wurden Zellstoffe mit erheblich höheren Festigkeiten hergestellt als bei hohen Aufschlußtemperaturen und kurzer Aufschlußdauer (Aufschluß A). 2. Bei niedriger Aufschlußtemperatur waren Ausbeute, Weißgrad und Aufschlußgrad der Zellstoffe geringer als bei hoher Aufschlußtemperatur. 3. Unter vergleichbaren Aufschlußbedingungen sind zwischen den Festigkeitseigenschaften von Zellstoffen unterschiedlicher Klone teilweise deutliche Unterschiede zu erkennen.

WKI-Kurzbericht Nr. 40/89

\section{NSSC-Halbzellstoffe aus dem Außen(Splint)- und Innen(Kern)-Bereich von Pappelholz}

B. Dix; E. Roffael, Wilhelm-Klauditz-Institut, Fraunhofer-Arbeitsgruppe für Hoizforschung Bienroder Weg $54 \mathrm{E}, 3300$ Braunschweig

Subject: Neutral Sulphite Semichemical (NSSC) pulps from the inner (heart) and outer (sap) wood of poplar.

Material und Methode: Von 16jährigem, entrindetem Pappelholz (Durchmesser ca. $18 \mathrm{~cm}$, Kernanteil ca. 15\%, Splintanteil ca. $85 \%$ ) des Klones Muhle-Larsen wurden Hackschnitzel aus dem Innen- $(r=3 \mathrm{~cm})$ und Außenbereich $(r=$ $6 \mathrm{~cm}$ ) hergestellt und nach dem NSSC-Verfahren aufgeschlossen. Maximale Aufschlußtemperatur: $170^{\circ} \mathrm{C}$, Aufheizdauer: $270 \mathrm{~min}$. Aufschlußdauer bei $170 \mathrm{C}: 60 \mathrm{~min}$, $\mathrm{Na}_{2} \mathrm{SO}_{3}$ /atro Holz: $14 \%, \mathrm{Na}_{2} \mathrm{CO}_{3} /$ atro Holz: $4 \%$, Flottenverhältnis (Holz: Lauge): 1:5. Aus den Halbzellstoffen wurden Prüfblätter hergestellt und deren wichtigsten physikalisch-technologischen Eigenschaften ermittelt. Zum Vergleich dienten Hackschnitzel, die über den gesamten Stamm hergestellt wurden.

\begin{tabular}{|c|c|c|c|c|}
\hline \multirow{2}{*}{$\begin{array}{l}\text { Zellstoff- } \\
\text { eigcn- } \\
\text { schaft }\end{array}$} & \multirow{2}{*}{$\begin{array}{l}\text { Ein- } \\
\text { heit }\end{array}$} & \multicolumn{3}{|l|}{ Zellstoff } \\
\hline & & $\begin{array}{l}\text { aus dem } \\
\text { Innen- } \\
\text { bereich }\end{array}$ & $\begin{array}{l}\text { aus dem } \\
\text { Außen- } \\
\text { bereich }\end{array}$ & $\begin{array}{l}\text { ïber den } \\
\text { Stamm- } \\
\text { quesschnitt }\end{array}$ \\
\hline Mahldauer & $\min$ & 15 & 15 & 15 \\
\hline Mahlgrad & SR & 29 & 28 & 26 \\
\hline Reißlänge & $\mathrm{km}$ & 7,87 & 8,14 & 7,60 \\
\hline $\begin{array}{l}\text { FortreiB- } \\
\text { festigkeit }\end{array}$ & $\frac{\mathrm{mN} \cdot \mathrm{m}}{\mathrm{m}}$ & 1045 & 1364 & 1262 \\
\hline Falzfestigkeit & - & 571 & 510 & 559 \\
\hline $\begin{array}{l}\text { Berst- } \\
\text { festigkejt }\end{array}$ & $\frac{\mathrm{kPa} \cdot \mathrm{m}^{2}}{\mathrm{~g}}$ & 4,32 & 4,37 & 4,15 \\
\hline Weißgrad & $\%$ & 41.6 & 46,1 & 46,0 \\
\hline Ligningehalt & $0 \%$ & 12,9 & 12,9 & 12,6 \\
\hline Ausbeule & $\%$ & 75,3 & 75,6 & 74.9 \\
\hline
\end{tabular}

Resultate: 1. Aus Hackschnitzeln des Außenbereiches von Pappelholz wurden Halbzellstoffe mit höherem Weißgrad und ebenso höherer Fortreißfestigkeit und Reißlänge hergestellt als aus Hackschnitzeln des Innenbereiches. 2. In der Ausbeute und im Ligningehalt bestanden zwischen den hergestellten Zellstoffen keine merklichen Unterschiede.

WKI-Kurzbericht Nr. 39/89 